\title{
Conflitos Éticos Vivenciados por Estudantes de Medicina
}

\section{Ethical Conflicts Experienced by Medical Students}

\section{PALAVRAS-CHAVE}

- Ética Médica.

- Bioética.

- Educação Médica.

- Estudantes de Medicina.

Recebido em: 03/10/2016

Aprovado em: 21/10/2016

\author{
Márcia Mendes Menezes ${ }^{I}$ \\ Luciana Colares Maia \\ Caroline Urias Rocha ${ }^{I}$ \\ Cristina Andrade Sampaio \\ Simone de Melo Costa
}

\section{RESUMO}

O atual estudo teve como objetivo identificar e analisar a prevalência de conflitos éticos vivenciados por estudantes de Medicina. Este estudo trata-se de pesquisa com delineamento transversal e analítico e que foi conduzida em uma escola pública do estado de Minas Gerais, Brasil. O instrumento utilizado para a coleta de dados foi um questionário autoaplicado. Os dados coletados foram apresentados em valores absolutos e percentuais. Para o tratamento estatístico analítico dos dados considerou-se o nível de significância $p<0,05$. As variáveis desfecho foram: Vivência de conflitos éticos em relações interpessoais no âmbito do curso médico e Condutas éticas na assistência em saúde. A identificação da prevalência dos conflitos éticos na graduação adotou a perspectiva das diferentes relações interpessoais (acadêmico-docente, acadêmico-acadêmico, acadêmico-funcionário, acadêmico-paciente, docente-docente, docente-paciente, docente-funcionário e funcionário-paciente) e condutas na assistência médica (importância de identificar-se ao usuário dos serviços de saúde e dele solicitar consentimento para realizar o exame físico, assistência sem supervisão do docente, emissão de documentos de saúde sem assinatura do profissional responsável e uso de redes sociais para compartilhar dados de paciente). Foi verificada associação das variáveis desfecho com sexo, ano da graduação e avaliação do curso. Participaram da pesquisa 281 acadêmicos matriculados em todos os anos da graduação em Medicina, de ambos os sexos, sendo predominante o sexo feminino(52,7\%). Os estudantes relataram ter vivenciado situações conflituosas nas relações interpessoais com os professores $(59,6 \%)$, prestaram assistência sem a devida supervisão de um professor (62,6\%), afirmaram ter emitido documentos de saúde sem o acompanhamento de professores (18,5\%), sendo a maior frequência observada entre aqueles matriculados nos anos mais avançados da graduação $(p<0,05)$. O uso das redes sociais com a finalidade de compartilhar os dados de pacientes $(25,1 \%$ ) foi prevalente nos anos mais avançados da graduação em medicina e entre os que avaliaram o curso como regular $(p<0,05)$. Conclui-se que os estudantes de graduação em Medicina vivenciaram conflitos éticos durante a sua formação médica, com prevalência de vivência dos conflitos nos anos mais avançados do referido curso. Nessa perspectiva, faz-se necessário propiciar um espaço de discussão e de reflexão coletiva acerca dos problemas éticos vivenciados pelos estudantes, ao longo da graduação em Medicina, a fim de se construir um agir profissional eticamente correto.
REVISTA BRASILEIRA DE EDUCAÇÃO MÉDICA 


\section{KEYWORDS}

- Medical Ethics.

- Bioethics.

- Medical Students.
- Medical Education.

ABSTRACT

The current study aimed to identify and analyze the prevalence of ethical conflicts experienced by medical students. This study is a cross-sectional and analytical research that was conducted in a public school in the state of Minas Gerais, Brazil. The instrument used for the data collection was a self-administered questionnaire. The data collected were presented in absolute and percentage values. For the analytical statistical treatment of the data, the level of significance was considered $p<0.05$. The outcome variables were: Experiences of ethical conflicts in interpersonal relations within the medical course and Ethical conduct in health care. The identification of the prevalence of ethical conflicts in the undergraduate program adopted the perspective of different interpersonal relations (academic-teaching, academic-academic, academic-employee, academic-patient, teacher-teacher, teacher-patient, teacher-employee and employee-patient). (Importance of identifying themselves to the health services user and requesting consent to perform the physical examination, assistance without the supervision of the teacher, issuance of health documents without the signature of the professional responsible and use of social networks to share data Of patient). It was verified the association of the outcome variables with sex, year of graduation and course evaluation. A total of 281 undergraduate students enrolled in all undergraduate courses in Medicine of both sexes, with a predominance of female (52.7\%). The students reported having experienced conflicting situations in interpersonal relations with teachers $(59.6 \%)$, provided assistance without proper supervision of a teacher $(62.6 \%)$, reported having issued health documents without the accompaniment of teachers (18,5\%). The highest frequency was observed among those enrolled in the most advanced years of the undergraduate program $(p<0.05)$. The use of social networks for the purpose of sharing patient data $(25.1 \%)$ was prevalent in the most advanced years of medical graduation and among those who evaluated the course as regular $(p<0.05)$. It is concluded that undergraduate medical students experienced ethical conflicts during their medical training, with a prevalence of conflicts in the advanced years of the course. From this perspective, it is necessary to provide a space for discussion and collective reflection on the ethical problems experienced by students, during their graduation in Medicine, in order to build a professional ethical practice.

\section{INTRODUÇÃO}

O Conselho Federal de Medicina, pelo Código de Ética Médica, no capítulo que trata dos Direitos Fundamentais,estabelece que cabe ao profissional zelar e trabalhar pelo excelente desempenho ético da medicina, pelo prestígio e pelo bom conceito da profissão. Tal aspecto sugere a necessidade de valorizar o conteúdo de ética na graduação ${ }^{1}$.

Com o desenvolvimento tecnológico na saúde, a descoberta de medicamentos e o uso de aparelhagens modernas surgiram outros valores no campo das relações interpessoais, afloraram conhecimentos científicos e colocaram-se em questão novos conflitos ${ }^{2}$. A formação ética dos futuros profissionais médicos é uma das responsabilidades da graduação, que deve proporcionar aos estudantes o desenvolvimento da capacidade de lidar com problemas no campo da moral, inerentes à prática profissional ${ }^{3}$.

Um dos instrumentos normativos internacionais, a Declaração sobre Bioética e Direitos Humanos, adotada pela Organização das Nações Unidas para a Educação, a Ciência e a
Cultura(Unesco), proclamou princípios éticos suscitados pela medicina, para orientar os Estados na construção de legislações e políticas. E destacou a educação, formação e informação em matéria de bioética como meio de assegurar a melhor compreensão das implicações éticas advindas dos progressos da ciência e tecnologia, em particular entre os jovens. Os Estados devem fomentar a educação e a formação bioética, em todos os níveis, e estimular a informação e difusão dos conhecimentos relacionados à bioética ${ }^{4}$.

A Declaração tem conformidade com o "Relatório Educação, um tesouro a descobrir", elaborado pela Comissão Internacional sobre Educação para o século XXI para a Unesco. O Relatório se fundamenta em quatro pilares do conhecimento: aprender a conhecer, aprender a fazer,aprender a viver juntos e aprender a ser. A Comissão considerou o aprender a ser a via essencial que integra os outros pilares do conhecimento. As políticas educativas servem como processo permanente para enriquecer conhecimentos do saber-fazer, contudo deveriam servir, em primeiro lugar, como uma via privilegiada para a 
construção da própria pessoa e das relações entre indivíduos e grupos ${ }^{5}$.

No pilar do conhecimento aprender a ser, a educação deve contribuir para o desenvolvimento da sensibilidade e responsabilidade pessoal. Todo ser humano deve ser preparado para elaborar pensamentos autônomos e críticos e formular seus próprios juízos de valores, de modo a decidir como agir nas diferentes circunstâncias da vida. Deve-se, na educação, fornecer forças e referências intelectuais para os jovens terem a compreensão do mundo e se comportarem nele como sujeitos atores responsáveis e justos. A escola deveria revalorizar a cultura oral e os conhecimentos advindos das experiências pessoais e não privilegiar o acesso ao conhecimento em detrimento de outras formas de aprendizagem, pois a educação deve ser concebida como um todo ${ }^{5}$.

As Diretrizes Curriculares Nacionais do curso de graduação em Medicina, no Brasil, destacaram a necessidade da formação ética e humanista dos graduados em Medicina, que deverão ser capazes de atuar com responsabilidade social e o compromisso de defender a cidadania, a dignidade humana e a saúde integral do ser humano ${ }^{6}$. Pouco se conhece sobre os conflitos éticos vividos no curso médico pelos graduandos. Nesse sentido, o objetivo deste estudo foi identificar e analisar a prevalência dos conflitos éticos vivenciados por estudantes de Medicina.

\section{MATERIAL E MÉTODO}

Trata-se de um estudo censitário, de cunho quantitativo, transversal e analítico, cuja população estudada foi composta por estudantes regularmente matriculados em um curso de graduação em Medicina, no Estado de Minas Gerais. De um potencial de 338 estudantes, 57 não retornaram o instrumento de coleta de dados, que foi um questionário semiestruturado e autoaplicado, elaborado pelos próprios autores com base na pesquisa bibliográfica sobre o tema: ética na formação médica. O questionário foi composto por questões acerca do perfil do estudante (período de matrícula, sexo e idade), percepções do sujeito com relação ao curso (avaliação do curso) e ética na formação médica (ensino da ética, Código de Ética Médica e do Estudante de Medicina e conflitos éticos vivenciados na graduação).

A identificação da prevalência dos conflitos éticos na graduação adotou a perspectiva das diferentes relações interpessoais (acadêmico-docente, acadêmico-acadêmico, acadêmico-funcionário, acadêmico-paciente, docente-docente, docente-paciente, docente-funcionário e funcionário-paciente) e condutas na assistência médica (importância de identificar-se ao usuário dos serviços de saúde e dele solicitar consentimento para realizar o exame físico, assistência sem supervisão do docente, emissão de documentos de saúde sem assinatura do profissional responsável e uso de redes sociais para compartilhar dados de paciente).

A aplicação do questionário foi efetuada após as atividades acadêmicas, tais como tutorias e palestras, após a avaliação da confiabilidade pelo teste-reteste conduzido com a participação de 12 estudantes, dois de cada ano do curso, com mesma proporção para mulheres e homens, no intervalo de 15 dias entre o teste e o reteste. A concordância das variáveis categóricas foi estimada pela estatística Kappa, sendo de substancial $(0,60$ a 0,80$)$ a quase perfeita $(0,80 \text { a } 1,00)^{7}$, demonstrando improvável dificuldade dos acadêmicos para interpretar as questões do instrumento de coleta de dados.

O tratamento estatístico dos dados foi conduzido no Programa IBM-SPSS 22.0, com realização da análise descritiva em valores percentuais e da análise bivariada pelo teste qui-quadrado de Pearson ou seu alternativo Fisher para associar os conflitos éticos na graduação com as variáveissexo, ano de graduação e avaliação do curso médico. Considerou-se o nível de significância igual a p<0,05.

Os resultados da pesquisa foram discutidos com base na pesquisa bibliográfica sobre o tema, com enfoque na abordagem reflexiva acerca dos conflitos éticos vivenciados na graduação em Medicina pelos estudantes.

O estudo foi conduzido de acordo com a Resolução 466/12 do Conselho Nacional de Saúde do Ministério da Saú$\mathrm{de}^{8}$. O projeto foi aprovado pelo Comitê de Ética em Pesquisa envolvendo seres humanos, sob o Parecer nº 845.561/2014. Cada participante recebeu e assinou um Termo de Consentimento Livre e Esclarecido, sendo garantido aos estudantes o anonimato e o sigilo das informações fornecidas, utilizadas exclusivamente para fins científicos.

\section{RESULTADOS}

O estudo contou com a participação de 281 alunos, o que representa $83,1 \%$ dos estudantes matriculados em todos os anos de graduação em Medicina, sendo a maioria referente aos graduandos dos três primeiros anos do curso (55,9\%) e de pessoas do sexo feminino (52,7\%). A taxa de não participação neste estudo foi de $16,9 \%$ dos estudantes, com proporções semelhantes entre os sexos.

O curso foi avaliado como muito bom para 30,8\% e bom para 54,5\%. Quanto ao ensino do conteúdo de ética na formação médica, considerou-se muito bom para 13,9\% e bom para 40,2\%. O conhecimento de boa parte do conteúdo do Código de Ética Médica do Conselho Federal de Medicina (CFM) foi destacado para $46,7 \%$ dos respondentes. A elaboração do Código de Ética do Estudante de Medicina específico para a instituição de sua graduação foi considerada importante pela maioria dos estudantes, $82,2 \%$ (Tabela 1 ). 
TABELA 1

Perfil do estudante de Medicina e considerações sobre a graduação e a ética médica

\begin{tabular}{|c|c|c|}
\hline Variáveis & n & $\%$ \\
\hline \multicolumn{3}{|l|}{ Sexo } \\
\hline Feminino & 148 & 52,7 \\
\hline Masculino & 133 & 47,3 \\
\hline \multicolumn{3}{|l|}{ Ano de matrícula na graduação } \\
\hline 1 a 3 & 157 & 55,9 \\
\hline 4 a 6 & 124 & 44,1 \\
\hline Total & 281 & 100,0 \\
\hline \multicolumn{3}{|l|}{ Avaliação do curso* } \\
\hline Muito bom & 86 & 30,8 \\
\hline Bom & 152 & 54,5 \\
\hline Regular & 41 & 14,7 \\
\hline Ruim & 0 & 0,0 \\
\hline Muito ruim & 0 & 0,0 \\
\hline \multicolumn{3}{|c|}{ Avaliação do ensino da ética na graduação } \\
\hline Muito bom & 39 & 13,9 \\
\hline Bom & 113 & 40,2 \\
\hline Regular & 99 & 35,2 \\
\hline Ruim & 23 & 8,2 \\
\hline Muito ruim & 7 & 2,5 \\
\hline Total & 281 & 100,0 \\
\hline \multicolumn{3}{|c|}{ Conhece o conteúdo do Código de Ética Médica?* } \\
\hline Completamente & 18 & 6,6 \\
\hline Boa parte & 128 & 46,7 \\
\hline Mais ou menos & 97 & 35,4 \\
\hline Pouco & 27 & 9,9 \\
\hline Muito pouco ou nada & 4 & 1,5 \\
\hline Total & 274 & 100,0 \\
\hline
\end{tabular}

Considera importante elaborar um Código de Ética do Estudante de Medicina?*

$\begin{array}{lrr}\text { Sim } & 226 & 82,2 \\ \text { Não } & 49 & 17,8 \\ \text { Total } & 275 & 100,0\end{array}$

* Perdas de respondentes às questões, valores percentuais ajustados.

TABELA 2

Conflitos éticos referidos em diferentes relações interpessoais na graduação em Medicina $(n=275)$

\begin{tabular}{|lrrrcr|}
\multicolumn{1}{c}{ Conflitos éticos } & \multicolumn{2}{c}{ Sim } & \multicolumn{2}{c|}{ Não } \\
\hline Relação interpessoal & n & \% & n & $\%$ \\
\hline Acadêmico-docente & 164 & 59,6 & 111 & 40,4 \\
\hline Acadêmico-acadêmico & 108 & 39,3 & 167 & 60,7 \\
\hline Acadêmico-paciente & 51 & 18,5 & 224 & 81,5 \\
\hline Acadêmico-funcionário & 36 & 13,1 & 239 & 86,9 \\
\hline Docente-paciente & 128 & 46,5 & 147 & 53,5 \\
\hline Docente-funcionário & 11 & 4,0 & 264 & 96,0 \\
\hline Docente-docente & 4 & 1,5 & 271 & 98,5 \\
\hline Paciente-funcionário & 11 & 4,0 & 264 & 96,0 \\
\hline
\end{tabular}

*Soma dos valores percentuais acima de 100,0\% pela identificação de conflitos éticos em mais de uma relação interpessoal.
Conflitos éticos nas relações interpessoais foram identificados, sendo a maior prevalência na relação entre acadêmico e docente $(59,6 \%)$ seguida da relação entre docente e paciente (46,5\%) e entre acadêmico e acadêmico (39,3\%) (Tabela 2).

A prevalência de conflito ético na relação interpessoal acadêmico e docente foi maior entre os estudantes dos anos mais avançados do curso médico (71,9\%) em comparação àqueles que cursam os primeiros anos da graduação $(\mathrm{p}<0,001)$. Os estudantes que avaliaram o curso como regular apresentaram maior prevalência de conflitos éticos na relação acadêmico e paciente $(41,5 \%)$ que aqueles com avaliação de muito bom/ bom $(14,2 \%)$, com significância estatística. Na relação conflituosa entre acadêmico e docente, as mulheres e os estudantes nos anos mais avançados foram os que apresentaram maior prevalência de conflitos vivenciados nessa relação interpessoal $(\mathrm{p}<0,05)$ (Tabela 3).

\begin{tabular}{|c|c|c|c|c|c|}
\hline \multicolumn{6}{|c|}{ TABELA 3} \\
\hline \multirow{3}{*}{\multicolumn{6}{|c|}{$\begin{array}{l}\text { Vivência de conflitos éticos em relações } \\
\text { interpessoais no âmbito do curso médico conforme } \\
\text { sexo, ano da graduação e avaliação do curso }\end{array}$}} \\
\hline & & & & & \\
\hline & & & & & \\
\hline \multicolumn{6}{|c|}{ Acadêmico-docente } \\
\hline \multirow[t]{2}{*}{ Variáveis } & \multicolumn{2}{|c|}{ Sim } & \multicolumn{2}{|c|}{ Não } & $\mathrm{p}$ \\
\hline & n & $\%$ & $\mathbf{n}$ & $\%$ & \\
\hline \multicolumn{6}{|l|}{ Sexo } \\
\hline Feminino & 88 & 61,1 & 56 & 38,9 & 0,601 \\
\hline Masculino & 76 & 58,0 & 55 & 42,0 & \\
\hline \multicolumn{5}{|l|}{ Ano da graduação } & $<0,001$ \\
\hline 1 a 3 & 77 & 50,0 & 77 & 50,0 & \\
\hline 4 a 6 & 87 & 71,9 & 34 & 28,1 & 0,206 \\
\hline \multicolumn{6}{|l|}{ Avaliação do curso* } \\
\hline Muito bom/bom & 134 & 57,8 & 98 & 42,2 & \\
\hline \multirow[t]{2}{*}{ Regular } & 28 & 68,3 & 13 & 31,7 & \\
\hline & \multicolumn{4}{|c|}{ Acadêmico-acadêmico } & \\
\hline \multirow{2}{*}{ Variáveis } & \multicolumn{2}{|c|}{$\operatorname{Sim}$} & \multicolumn{2}{|c|}{ Não } & $\mathrm{p}$ \\
\hline & $\mathbf{n}$ & $\%$ & $\mathbf{n}$ & $\%$ & \\
\hline \multicolumn{6}{|l|}{ Sexo } \\
\hline Feminino & 52 & 36,1 & 92 & 63,9 & 0,260 \\
\hline Masculino & 56 & 42,7 & 75 & 57,3 & \\
\hline \multicolumn{5}{|l|}{ Ano da graduação } & 0,905 \\
\hline 1 a 3 & 60 & 39,0 & 94 & 61,0 & \\
\hline 4 a 6 & 48 & 39,7 & 73 & 60,3 & 0,173 \\
\hline \multicolumn{6}{|l|}{ Avaliação do curso* } \\
\hline Muito bom/bom & 87 & 37,5 & 145 & 62,5 & \\
\hline \multirow[t]{2}{*}{ Regular } & 20 & 48,8 & 21 & 51,2 & \\
\hline & \multicolumn{4}{|c|}{ Acadêmico-paciente } & \\
\hline \multirow[t]{2}{*}{ Variáveis } & \multicolumn{2}{|c|}{ Sim } & \multicolumn{2}{|c|}{ Não } & $\mathrm{p}$ \\
\hline & $\mathbf{n}$ & $\%$ & $\mathbf{n}$ & $\%$ & \\
\hline \multicolumn{6}{|l|}{ Sexo } \\
\hline Feminino & 29 & 20,1 & 115 & 79,5 & 0,476 \\
\hline Masculino & 22 & 16,8 & 109 & 83,2 & \\
\hline Ano da graduação & & & & & 0,154 \\
\hline 1 a 3 & 24 & 15,6 & 130 & 84,4 & \\
\hline 4 a 6 & 27 & 22,3 & 94 & 77,7 & $<0,001$ \\
\hline \multicolumn{6}{|l|}{ Avaliação do curso* } \\
\hline Muito bom/bom & 33 & 14,2 & 199 & 85,8 & \\
\hline Regular & 17 & 41,5 & 24 & 58,5 & \\
\hline
\end{tabular}




\begin{tabular}{|c|c|c|c|c|c|}
\hline \multirow{3}{*}{ Variáveis } & \multicolumn{4}{|c|}{ Docente-paciente } & \multirow{3}{*}{ p } \\
\hline & \multicolumn{2}{|c|}{ Sim } & \multicolumn{2}{|c|}{ Não } & \\
\hline & $\mathbf{n}$ & $\%$ & $\mathrm{n}$ & $\%$ & \\
\hline \multicolumn{6}{|l|}{ Sexo } \\
\hline Feminino & 76 & 52,8 & 68 & 47,2 & 0,030 \\
\hline Masculino & 52 & 39,7 & 79 & 60,3 & \\
\hline Ano da graduação & & & & & $<0,001$ \\
\hline 1 a 3 & 50 & 32,5 & 104 & 67,5 & \\
\hline 4 a 6 & 78 & 64,5 & 43 & 35,5 & 0,513 \\
\hline \multicolumn{6}{|l|}{ Avaliação do curso* } \\
\hline Muito bom/bom & 109 & 47,0 & 123 & 53,0 & \\
\hline Regular & 17 & 41,5 & 24 & 58,5 & \\
\hline
\end{tabular}

* Perdas de respondentes às questões, valores percentuais ajustados.

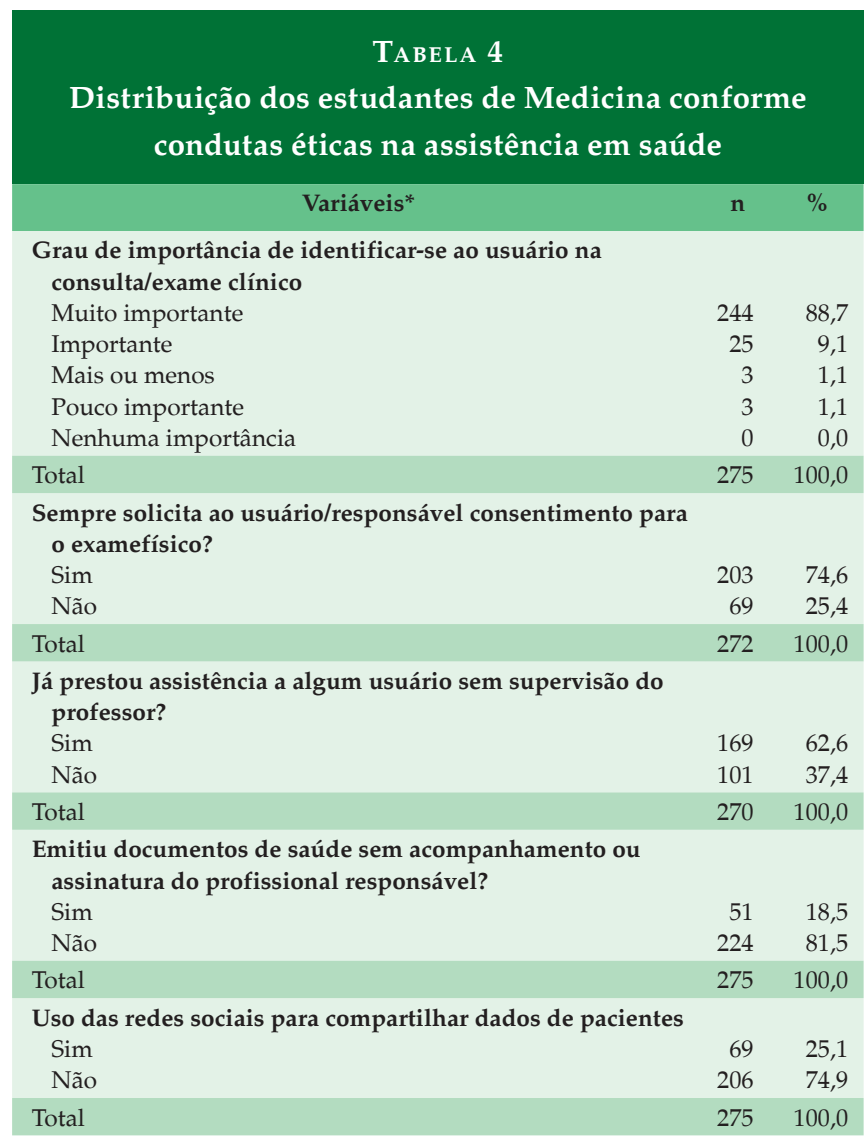

* Perdas de respondentes às questões, valores percentuais ajustados.

A necessidade de identificar-se ao usuário dos serviços de saúde durante o atendimento clínico foi considerada muito importante por $88,7 \%$ dos estudantes, sendo que $74,6 \%$ sempre solicitam consentimento para a realização do exame físico ao paciente ou responsável. A prestação de assistência a algum usuário sem a supervisão do profissional responsável foi destacada por $62,6 \%$ dos estudantes, e a emissão de algum documento de saúde, como receitas, prescrições e/ou atestados, no ato da assistência, sem o acompanhamento e assinatura do docente foi relatada por $18,5 \%$ dos graduandos. $\mathrm{O}$ uso das redes sociais para enviar fotos de situações clínicas ou para discutir informações de pacientes com colegas, docentes ou amigos foi confirmado por $25,1 \%$ dos universitários (Tabela 4).

Identificar-se ao usuário dos serviços de saúde foi considerado muito importante/importante em maior percentual $(98,7 \%)$ entre os estudantes que melhor classificaram o curso médico $(\mathrm{p}<0,05)$. A solicitação do consentimento ao paciente para o exame físico foi mais prevalente entre os acadêmicos dos primeiros anos da graduação $(85,4 \%)$, com diferença significativa. Diferentemente do resultado anterior, a assistência e a emissão de documentos de saúde sem supervisão profissional foram maiores entre os que se encontravam nos anos mais avançados da graduação $(p<0,05)$. Compartilhar informações de pacientes nas redes sociais não foi associado ao sexo, apesar de os homens terem feito maior uso dessa tecnologia $(27,7 \%)$ que as mulheres $(22,8 \%), p=0,346$. Estar matriculado nos três últimos anos da graduação e classificar o curso médico como regular mantiveram-se associados ao ato de utilizar as redes sociais na assistência à saúde (Tabela 5).

\begin{tabular}{|c|c|c|c|c|c|}
\hline \multicolumn{6}{|c|}{$\begin{array}{l}\text { Condutas na assistência em saúde conforme } \\
\text { sexo, ano da graduação e avaliação do curso } \\
\text { médico pelos estudantes de Medicina }\end{array}$} \\
\hline \multirow{3}{*}{ Variáveis } & \multicolumn{4}{|c|}{ Identificar-se ao usuário de saúde } & \multirow{3}{*}{ p } \\
\hline & \multicolumn{2}{|c|}{$\begin{array}{l}\text { Muito importante/ } \\
\text { importante }\end{array}$} & \multicolumn{2}{|c|}{$\begin{array}{l}\text { Mais ou menos/ } \\
\text { pouco importante }\end{array}$} & \\
\hline & $\mathrm{n}$ & $\%$ & $\mathbf{n}$ & $\%$ & \\
\hline \multicolumn{6}{|l|}{ Sexo } \\
\hline Feminino & 143 & 98,6 & 2 & 1,4 & 0,426 \\
\hline Masculino & 126 & 96,9 & 4 & 3,1 & \\
\hline Ano da graduação & & & & & 0,411 \\
\hline 1 a 3 & 151 & 98,7 & 2 & 1,3 & \\
\hline 4 a 6 & 118 & 96,7 & 4 & 3,3 & 0,046 \\
\hline \multicolumn{6}{|l|}{ Avaliação do curso } \\
\hline Muito bom/bom & 229 & 98,7 & 3 & 1,3 & \\
\hline \multirow[t]{2}{*}{ Regular } & 38 & 92,7 & 3 & 7,3 & \\
\hline & \multicolumn{4}{|c|}{$\begin{array}{c}\text { Consentimento do usuário para o } \\
\text { exame físico }\end{array}$} & \\
\hline \multirow[t]{2}{*}{ Variáveis } & \multicolumn{2}{|c|}{ Sim } & \multicolumn{2}{|c|}{ Não } & $\mathrm{p}$ \\
\hline & $\mathbf{n}$ & $\%$ & $\mathbf{n}$ & $\%$ & \\
\hline \multicolumn{6}{|l|}{ Sexo } \\
\hline Feminino & 102 & 71,3 & 41 & 28,7 & 0,187 \\
\hline Masculino & 101 & 78,3 & 28 & 21,7 & \\
\hline Ano da graduação & & & & & $<0,001$ \\
\hline 1 a 3 & 129 & 85,4 & 22 & 14,6 & \\
\hline 4 a 6 & 74 & 61,2 & 47 & 38,8 & 0,473 \\
\hline \multicolumn{6}{|l|}{ Avaliação do curso } \\
\hline Muito bom/bom & 174 & 76,0 & 55 & 24,0 & \\
\hline Regular & 29 & 70,7 & 12 & 29,3 & \\
\hline
\end{tabular}




\begin{tabular}{|c|c|c|c|c|c|}
\hline \multicolumn{6}{|c|}{$\begin{array}{l}\text { Condutas na assistência em saúde conforme } \\
\text { sexo, ano da graduação e avaliação do curso } \\
\text { médico pelos estudantes de Medicina }\end{array}$} \\
\hline \multirow{3}{*}{ Variáveis } & \multicolumn{4}{|c|}{ Assistência sem supervisão } & \\
\hline & \multicolumn{2}{|c|}{ Sim } & \multicolumn{2}{|c|}{ Não } & $\mathrm{p}$ \\
\hline & n & $\%$ & $\mathrm{n}$ & $\%$ & \\
\hline \multicolumn{6}{|l|}{ Sexo } \\
\hline Feminino & 88 & 60,7 & 57 & 39,3 & 0,486 \\
\hline Masculino & 81 & 64,8 & 44 & 35,2 & \\
\hline Ano da graduação & & & & & 0,004 \\
\hline 1 a 3 & 82 & 55,0 & 67 & 45,0 & \\
\hline 4 a 6 & 87 & 71,9 & 34 & 28,1 & 0,118 \\
\hline \multicolumn{6}{|l|}{ Avaliação do curso } \\
\hline Muito bom/bom & 139 & 60,4 & 91 & 39,6 & \\
\hline Regular & 28 & 73,7 & 10 & 26,3 & \\
\hline \multicolumn{6}{|c|}{$\begin{array}{l}\text { Emissão de documentos sem } \\
\text { supervisão }\end{array}$} \\
\hline \multirow[t]{2}{*}{ Variáveis } & \multicolumn{2}{|c|}{ Sim } & \multicolumn{2}{|c|}{ Não } & $\mathrm{p}$ \\
\hline & n & $\%$ & $\mathrm{n}$ & $\%$ & \\
\hline \multicolumn{6}{|l|}{ Sexo } \\
\hline Feminino & 24 & 16,6 & 121 & 83,4 & 0,369 \\
\hline Masculino & 27 & 20,8 & 103 & 79,2 & \\
\hline Ano da graduação & & & & & $<0,001$ \\
\hline 1 a 3 & 10 & 6,5 & 143 & 93,5 & \\
\hline 4 a 6 & 41 & 33,6 & 81 & 66,4 & 0,777 \\
\hline \multicolumn{6}{|l|}{ Avaliação do curso } \\
\hline Muito bom/bom & 41 & 17,7 & 191 & 82,3 & \\
\hline Regular & 8 & 19,5 & 33 & 80,5 & \\
\hline \multirow{3}{*}{ Variáveis } & \multicolumn{4}{|c|}{$\begin{array}{l}\text { Compartilhou dados de pacientes } \\
\text { nas redes sociais/internet? }\end{array}$} & \\
\hline & Sim & & Não & & $\mathrm{p}$ \\
\hline & $\mathbf{n}$ & $\%$ & $\mathrm{n}$ & $\%$ & \\
\hline \multicolumn{6}{|l|}{ Sexo } \\
\hline Feminino & 33 & 22,8 & 112 & 77,2 & 0,346 \\
\hline Masculino & 36 & 27,7 & 94 & 72,3 & \\
\hline Ano da graduação & & & & & $<0,001$ \\
\hline 1 a 3 & 24 & 15,7 & 129 & 84,3 & \\
\hline 4 a 6 & 45 & 36,9 & 77 & 63,1 & 0,023 \\
\hline \multicolumn{6}{|l|}{ Avaliação do curso } \\
\hline Muito bom/bom & 52 & 22,4 & 180 & 77,6 & \\
\hline Regular & 16 & 39,0 & 25 & 61,0 & \\
\hline
\end{tabular}

\section{DISCUSSÃO}

Neste estudo foi observada predominância do sexo feminino entre os estudantes de Medicina, dado coerente com um estudo que apresentou o fenômeno da feminilização da saúde, inclusive no curso médico?.

Apesar de o conteúdo de ética ser trabalhado principalmente em dois módulos, no primeiro e quarto ano, a avaliação da abordagem do ensino do conteúdo de ética foi muito boa e boa, na visão da maioria dos estudantes. O estudo da ética profissional e bioética deve constituir uma das prioridades na formação médica, aliado ao conhecimento técnico, uma vez que o futuro profissional se ocupará do cuidado da saúde de pessoas ${ }^{10,11}$.

Quanto ao conhecimento do conteúdo do Código de Ética Médica do Conselho Federal de Medicina (CFM), a maioria conhece boa parte/completamente. Resultado importante, uma vez que esse documento rege a conduta dos profissionais médicos, e os acadêmicos necessitam desses saberes, pois no futuro estarão sujeitos à fiscalização do Conselho de classe ${ }^{12}$.

Os estudantes consideraram importante a elaboração do Código de Ética do Estudante de Medicina(Ceem), específico para a instituição de sua graduação. A importância de tal Código vai além do estímulo à prevenção do exercício ilegal da profissão. Incide, também, na humanização do futuro médico, que por meio desse instrumento absorverá os princípios e diretrizes voltados ao exercício cidadão da medicina ${ }^{13}$.

A existência de conflitos éticos nas relações interpessoais foi destacada pelos graduandos, com maior prevalência na relação docente-acadêmico entre os matriculados nos últimos anos do curso. Na relação docente e paciente, os conflitos éticos foram mais vivenciados pelas mulheres e também entre aqueles em estágio mais avançado da graduação. Esse resultado pode ser explicado, em parte, pela maior sensibilidade das mulheres na percepção das situações eticamente conflituosas e pelo maior tempo vivido na graduação.

Os conflitos na relação acadêmico e paciente foram mais prevalentes entre os que avaliaram o curso como regular. $\mathrm{Na}$ relação estudante e estudante, os conflitos vivenciados, por mais de um terço dos estudantes, não foram associados a sexo, ano de graduação e avaliação do curso médico.

Deve-se respeitar a dignidade humana, os direitos dos seres humanos e as liberdades fundamentais. ${ }^{4}$. Os pilares do conhecimento aprender a viver juntos e aprender a viver com os outros representam um dos maiores desafios da educação. Ensinar a não violência nas instituições de ensino é uma ideia de luta contra os preconceitos geradores de conflitos. As pessoas tendem a supervalorizar suas qualidades e as de seu grupo e a alimentar preconceitos em relação aos outros, exacerbando as rivalidades históricas. Para minimizar a violência, a educação deve utilizar vias complementares, a descoberta progressiva do outro, que seria o primeiro nível, e, ao longo de toda a vida, no segundo nível, estimular a participação em projetos comuns entre os diferentes sujeitos para evitar ou resolver conflitos latentes ${ }^{5}$.Cabe à escola médica proporcionar aos estudantes o desenvolvimento da capacidade de lidar com problemas no campo da moral, com que eles se defrontarão durante a prática profissional ${ }^{3}$. 
Os acadêmicos consideraram importante identificar-se ao usuário dos serviços de saúde durante o atendimento clínico e ser necessário obter o consentimento para realizar o exame físico na assistência em saúde. Atitudes eticamente corretas e que devem ser estimuladas pelos professores ao longo da graduação em Medicina, pois neste estudo observou-se maior frequência dessa atitude nos estudantes dos períodos iniciais.

$\mathrm{O}$ estudante deve aprender que o ser humano merece e precisa ser respeitado e não considerado apenas como mero objeto de estudo. Infelizmente, há certa banalização do desrespeito ao indivíduo em muitos ambientes de prática profissional, fruto da "coisificação" do ser humano ${ }^{3}$. O consentimento é um princípio ético que deve ser respeitado, sendo que qualquer intervenção na área médica, seja de caráter preventivo, diagnóstico ou terapêutico, só poderá ser realizada com o prévio consentimento livre e esclarecido do sujeito ${ }^{4}$.

A prestação de assistência sem supervisão do docente foi destacada por grande parte dos estudantes, com maior prevalência para os matriculados nos períodos finais do curso. Deve-se considerar que, com o auxílio da supervisão docente, os estudantes adquirem maior autoconfiança e se tornam mais capacitados a estabelecer relações autênticas com os pacientes e a assumir maior responsabilidade no cuidado dos mesmos ${ }^{14}$.

A emissão de documentos de saúde, como receitas, prescrições e/ou atestados sem o acompanhamento e assinatura do docente/profissional responsável é algo que ocorre no âmbito da formação em Medicina, com maior prevalência para os matriculados nos anos finais do curso.

É necessário interrogar esse papel de formação em que o supervisor deveria orientar o estudante nos aspectos tanto técnicos como éticos ${ }^{15}$ e ter o compromisso de acompanhar e supervisionar as atitudes dos graduandos. Entre as características de um bom docente enquadra-se o respeito aos princípios éticos no âmbito da profissão médica.

Nessa perspectiva, estudos destacaram características importantes para classificar positivamente o docente. Entre elas, a ética foi sinalizada juntamente com outras características técnicas, didáticas e científicas ${ }^{16-19}$.

No atual estudo, confirmou-se o uso das redes sociais para compartilhar fotos de situações clínicas ou para discutir informações de pacientes, sendo a maior prevalência entre os estudantes dos últimos anos de graduação e entre os que consideraram o curso como regular. Trata-se de uma situação eticamente conflituosa vivenciada pelos estudantes, pois se considera direito do paciente ter sua privacidade preservada,e dever do médico orientar seus auxiliares e graduandos a zelar pela manutenção do sigilo profissional ${ }^{1}$.A vida privada dos sujeitos e a confidencialidade de suas informações devem ser respeitadas. Não se deve utilizar ou difundir informações para outros fins que não aqueles para os quais foram coligidos ou consentidos, que, mesmo assim, devem estar em consonância com o direito internacional relativo aos direitos humanos ${ }^{4}$.

Ainda no Código de Ética Médica, no capítulo IX e artigo 75 , veda-se ao médico fazer referência a casos clínicos identificáveis, exibir pacientes ou seus retratos em anúncios profissionais ou na divulgação de assuntos médicos em meios de comunicação em geral, mesmo com autorização do paciente ${ }^{1}$. Nessa perspectiva, é necessário que professores estejam atentos a essas atitudes dos estudantes, futuros profissionais médicos, e os orientem quanto ao uso das redes sociais de forma adequada no exercício da medicina. A quebra do sigilo profissional é atitude considerada infração ética, com previsão de penalidades pelo Conselho Federal de Medicina. Expor fatos e imagens de pacientes nas redes sociais poderá implicar prejuízos para a carreira profissional e constrangimentos ou prejuízos ao paciente.

Como limitações, este estudo tem delineamento transversal e por isso as associações entre variáveis não podem ser interpretadas como relação de causa e efeito. Devem-se considerar os possíveis vieses de informação e de memória, uma vez que os dados foram coletados por meio de um questionário autoaplicado, e os estudantes podem não ter registrado todos os conflitos éticos vivenciados na graduação.

$\mathrm{O}$ atual estudo permitiu refletir acerca dos conflitos éticos vivenciados pelos estudantes no âmbito da graduação. Entre eles, destaca-se o compartilhamento de dados de pacientes nas redes sociais, o que caracteriza quebra da privacidade e do anonimato inerentes ao ato médico. Grande parte dos conflitos relacionados à assistência em saúde foi associada aos anos mais avançados da graduação. Assim, devem-se adotar estratégias na instituição para propiciar um espaço de discussão e reflexão coletiva acerca dos problemas ao longo da graduação, em busca de resolução ou, pelo menos, minimização das situações conflituosas vivenciadas pelos estudantes de Medicina e melhor construção do agir profissional eticamente correto. O diálogo cotidiano deve fazer parte da construção de seres humanos éticos e de graduandos cidadãos.

\section{Apoio}

Fundação de Amparo à Pesquisa do Estado de Minas Gerais (Fapemig). Processo $n^{\circ}$ CHE-APQ. 00707-15 e bolsa de pesquisa Processo BIP 00058-16.

\section{REFERÊNCIAS}

1. CFM. Código de Ética Médica. Conselho Federal de Medicina. Brasília-DF: CFM; 2010. 
2. Roque MV. A relação pessoal como acesso privilegiado para o doente mental grave. Rev Bioét 2001; 9(1): 53-62.

3. Rego SA. A formação ética dos médicos: saindo da adolescência com a vida (dos outros) nas mãos. Rio de Janeiro: Fiocruz; 2003.

4. UNESCO. Organização das Nações Unidas para a Educação, Ciência e Cultura [on line]. Declaração Universal sobre Bioética e Direitos Humanos. 2005. [capturado 27 set. 2015]. Disponível em: http://unesdoc.unesco.org/ images/0014/001461/146180por

5. Delors J, Al-Mufti I, Amagi I, Carneiro R, Chung F, Geremek B, et al. Educação um tesouro a descobrir. Relatório para a UNESCO da Comissão Internacional sobre Educação para o século XXI. São Paulo: Cortez Editora; 1997.

6. Brasil. Conselho Nacional de Educação. Resolução 03/2014 [on line]. Institui Diretrizes Curriculares Nacionais do Curso de Graduação em Medicina e dá outras Providências [capturado 20 ago. 2015]. 2014. Disponível em: http://portal.mec.gov.br/index.php?option=com docman\&view =download \&alias $=15874-$ rces003-14\&category_slug=junho-2014-pdf\&Itemid=30192

7. Griep RH, Chor D, Faerstein E, Lopes C. Confiabilidade teste-resteste de aspectos da rede social no Estudo Pró-saúde. Rev. Saúde Pública. 2003; 37(3):379-385.

8. Brasil.Ministério da Saúde. Conselho Nacional de Saúde. Resolução CNS 466/2012. Aprova diretrizes e normas regulamentadoras de pesquisa envolvendo seres humanos. Brasília: Conselho Nacional de Saúde; 2012.

9. Scheffer MC, Cassenote AJF. A feminização da medicina no Brasil. Rev Bioét. 2013; 21(2): 268-277.

10. Gomes JCM. O atual ensino da ética para os profissionais de saúde e seus reflexos no cotidiano do povo brasileiro. Rev Bioét. 1996; 4(1):53-64.

11. Gomes AMA, Moura ERF, Amorim RF. O lugar da Ética e Bioética nos currículos de Formação Médica. Rev bras educ méd. 2006; 30(2):56-65.

12. Almeida AM, Bitencourt AGV, Neves NMBC, Neves FBCS, Lordelo MR, Lemos KM, Nuñez, GR, Barbetta MC, Athanazio RA, Nery-Filho A. Conhecimento e Interesse em Ética Médica e Bioética na Graduação Médica. Rev bras educ méd 2008; 32(4):437-444.

13. Lisboa L, Lins L. Código de ética do estudante de medicina: uma análise qualitativa. Rev Bioét. 2014; 22(1):182-190.
14. Botti SHO, Rego, S. Preceptor, supervisor, tutor e mentor: quais são seus papéis? Rev bras educ méd. 2008; 32(3):363373.

15. Taquette SR, Rego S, Schramm FR, Soares LL, Carvalho SV. Situações eticamente conflituosas vivenciadas por estudantes de medicina. Rev Assoc Med Bras. 2005; 51(1): 23-28.

16. Knight LV, Bligh J. Physicians perceptions of clinical teaching: a qualitative analysis in the context of change. Adv Health Sci Educ Theory Pract. 2006; 11(3):221-234.

17. Sutkin G, Wagner E, Harris I, Schiffer R. What makes a good clinical teacher in medicine? A review of the literature. Acad Med 2008; 83(5):452-466.

18. Duvivier RJ, Van Dalen J, Van Der Vleuten COM, Scherpbier AJJA. Teacher perceptions of desired qualities, competencies and strategies for clinical skills teachers. Med Teach 2009; 31(7):634-641.

19. Stenfors-Hayes T, Hult H, Dahlgren LO. What does it mean to be a good teacher and clinical supervisor in medical education? Adv Health Sci Educ Theory Pract. 2011; 16(2): 197-210.

\section{CONTRIBUIÇÃO DOS AUTORES}

Márcia Mendes Menezes e Simone de Melo Costa participaram de todas as etapas do estudo: planejamento, execução, redação e revisão final do manuscrito. Caroline Urias Rocha participou da execução. Cristina Andrade Sampaio e Luciana Colares Maia contribuíram na execução e revisão final do manuscrito.

\section{CONFLITO DE INTERESSES}

Não há conflito de interesses.

\section{ENDEREÇO PARA CORRESPONDÊNCIA}

Simone de Melo Costa

Universidade Estadual de Montes Claros

Avenida Dr. Rui Braga, s/nº-prédio 6 - sala 109

Campus Universitário Prof. Darcy Ribeiro-Montes Claros

CEP 39401-089 - MG

E-mail: marciam.menezes@yahoo.com.br; luciana_colares@ yahoo.com.br; carolurocha@hotmail.com; sampaio.cristina@ uol.com.br; smelocosta@gmail.com 